\title{
Perú: liderazgo y toma de decisiones en el momento actual
}

\author{
CPC Julio Palomino Silva
}

Docente Auxiliar de la Facultad de Ciencias Contables

\section{RESUMEN}

El Perú vive un momento especial en su historia económica, al crecimiento sostenible en los últimos años, se suma la reunión del XVI Foro de Cooperación Económica Asia - Pacifico (APEC) en nuestro país, una vitrina mundial para exponer nuestras potenciales riquezas, parece que los peruanos hemos comprendido que tenemos una gran oportunidad que no podemos desaprovechar, el liderazgo empresarial es importante para enfrentar los nuevos retos y poder tomar decisiones que nos conduzcan al éxito. El buen liderazgo y la buena toma de decisiones van de la mano. El liderazgo eficiente depende de la capacidad de saber cuándo avanzar y cuándo cambiar de curso, además de la capacidad de demostrar propósito y dirección. Esto resulta esencial cuando se moviliza a la gente, se determinan prioridades y se genera compromiso.

Este Artículo trata de describir el rol del liderazgo y el compromiso de los lideres para garantizar que las decisiones estratégicas se desarrollen y se implementen con eficiencia. También proporciona pautas para lograrlo y un resumen de las ideas y los temas principales que hay detrás de este proceso de toma de decisiones.

Palabras clave: Liderazgo, empresa, innovación, creatividad, decisiones. 


\section{INTRODUCCIÓN}

El liderazgo es un tema crucial hoy en día en donde las fronteras se han abierto al comercio global; donde las organizaciones y las empresas permanentemente se encuentran en una constante lucha por ser cada vez más competitivas, lo que ha generado que las personas que la conforman sean eficientes y capaces de dar mucho de sí para el bienestar de la organización y la empresa. Hoy en momentos cruciales para el Perú, se necesitan de líderes capaces de forjar nuevas generaciones con una nueva mentalidad gerencial y que permita aprovechar las oportunidades que se presenten, mediante una buena toma de decisiones.

\section{El liderazgo decisivo}

La toma de decisiones eficiente depende de una serie de destrezas de liderazgo que se pueden aprender y que a menudo están estrechamente vinculadas. He aquí algunas:

- La capacidad de fomentar la innovación y la creatividad y de aprovechar las sinergias entre las personas a veces entre equipos dispares y distantes.

- La inteligencia y el coraje de reconocer los errores y aprender de ellos, capacidad de ayudar a otros a hallar sus soluciones.

- Habilidad para delegar de forma tal que la toma de decisiones pueda ser transferida a otros integrantes de la organización con suficiente tiempo o información.

- La capacidad de motivar a las personas para que tengan la inspiración de prevenir o resolver los problemas por sí mismas, además de implementar las decisiones en forma preactiva.

- La capacidad de enfocar a otros en las cuestiones igualmente importantes de servir a los clientes y gestionen los cambios.
- Las habilidades de comunicación.

- El coraje y la capacidad de tomar decisiones críticas.

\section{Fomentar la innovación y la creatividad}

A muchas personas les cuesta hallar nuevas ideas o soluciones para un problema. La respuesta suele encontrarse en fomentar la innovación, creando las condiciones propicias para ser innovadores o, simplemente, para ver la mejor manera de avanzar. Para que esto dé resultado y sea sostenible, las decisiones se deben tomar en un clima lo más positivo, abierto y alentador posible. Esto guarda relación con la necesidad de la gente de sentirse valorada. Si no se siente así, existe el riesgo de que haya reacciones al cambio en forma negativa, de que sea reactiva más que preactiva. $\mathrm{O}$ incluso de que decida marcharse.

Una manera de fomentar la innovación consiste en alentar a los empleados a cuestionar la manera en que se hacen las cosas o las decisiones que se han tomado. El hecho de eliminar o minimizar barreras (como la burocracia o las jerarquías) puede impulsar la innovación, igual que la capacidad de ejercer una presión moderada, quizás estableciendo plazos. Alentar la innovación significa escuchar las sugerencias con mente abierta.

Esto es importante, porque si la gente siente que ha participado en una decisión, que ha sido tomada con inteligencia y sensibilidad, apoyará esa decisión y su implementación. Si se escuchan las opiniones y sugerencias de la gente y se las toma en cuenta, la decisión final debería ser, al menos en teoría mejor.

\section{Aprender de los errores}

Cometer errores es la clave para avanzar. Los errores no son solo excelentes oportunida- 
des de aprender, también son, en un sentido, importantes. La única oportunidad de aprender algo nuevo. La evolución biológica avanza mediante por un inmenso e inexorable proceso de prueba y error, y sin los errores, las pruebas no lograrían nada.

Aprender de los errores, además, pone de manifiesto otro aspecto importante del liderazgo decisivo: el coraje y la capacidad de actuar solos.

Una tendencia natural de evaluar el presente o de concentrarse en la decisión inmediata debería atemperarse con cierta perspectiva futura y pasada. Es necesario tratar de no mal interpretar las lecciones del pasado ni utilizar los acontecimientos para justificar las decisiones actuales pero con una lógica espuria. Las trampas de la inmovilidad, del costo hundido y de la evidencia confirmada son todas fallas de conducta que se basan en actitudes específicas respecto de los hechos pasados. Es crucial que haya una comprensión genuina del pasado, pero debe estar relacionada con lo que ocurre en el presente y mantenerse en perspectiva.

Cuanto más alto trepamos, más abarca la vista. Cada nuevo punto de observación brinda una mejor comprensión de la interconexión de las cosas. Lo que es más, la acumulación gradual de comprensión se ve salpicada por súbitas y sorprendentes ampliaciones del horizonte, como cuando llegamos a la cima de una colina y vemos cosas que nunca imaginamos durante la escalada. Una vez que nos hemos orientado en el nuevo paisaje, nuestro sendero a la cima recién alcanzada queda despejado y toma su sitio honorable en el nuevo mundo.

\section{Análisis de opciones contrarias}

Es común que en la toma de decisiones no haya un análisis suficientemente exhaustivo.
La tendencia natural es que la gente gravite hacia una opción en particular, a menudo por prejuicio, temor (especialmente temor al cambio), deseo de evitar riesgos, pereza o por basarse demasiado en el instinto. Es importante cuestionar las suposiciones y ver las cosas desde otras perspectiva (como la del cliente o la de la persona que se ve más afectada por la decisión).

Una manera de encarar las opciones contrarias consiste en mirar el resultado deseado y luego ver cuál de las opciones servirá para lograrlo. Este enfoque sencillo suele complicarse por la necesidad de priorizar las metas, reducir costos o minimizar los riesgos, y para seleccionar la mejor opción a menudo hay que hacer concesiones. Otro enfoque consiste en establecer criterios para la decisión final, por ejemplo, que es necesario que dé resultados con rapidez, que no sea costosa, que su organización insuma una cantidad razonable de tiempo, y así sucesivamente - y luego calificar cada opción en virtud de estos componentes según una escala del 1 al 10 . La opción que obtenga el puntaje más alto, gana-. Con ambos enfoques, es necesario entender cabalmente qué requiere cada opción, como funciona y qué se logra con ella.

\section{Delegar}

Con frecuencia, el éxito o el fracaso de una decisión dependen del proceso de delegación. El hecho de facultar a otros (empowerment), va más allá de la delegación de tareas específicas, implica otorgar un nivel definido de autoridad y responsabilidad dentro del cual alguien toma sus propias decisiones y las implementa.

La delegación de poder (o empowerment), es una verdadera apertura del poder creativo del personal. Se basa en la creencia de que a menudo no se aprovecha toda la 
capacidad de los empleados y que, si se les da una oportunidad y la responsabilidad, ellos desean hacer un aporte positivo.

La delegación de poder va más allá de la delegación y es una manera de permitir que la gente aproveche más plenamente su potencial. En esencia, significa permitir que los individuos hagan su trabajo, alentando a quienes están más cerca de la acción a tomar sus propias decisiones. Se necesita apoyo, confianza y voluntad de retirar los obstáculos y las burocracias, alentando y permitiendo a la gente que lleve a la práctica sus ideas para mejorar.

A fin de facultar a la gente para tomar decisiones y luego implementarlas, es necesario que los líderes:

- Establezcan un rumbo claro y se aseguren de que la gente no se desvíe del curso.

- Alienten a la gente a ser innovadora y aprovechar su iniciativa, por lo general dentro de límites acordados.

- Retengan una plena comprensión de los que está ocurriendo.

- Creen un ambiente positivo, de apoyo y sin culpas.

- Apoyen con frecuencia y despejen la vía de acción sin quitar responsabilidades a quienes están haciendo el trabajo.

- Tomen decisiones que otros no pueden tomar, ya sea por falta de tiempo, información o conocimiento.

- Evalúen continuamente el desempeño y recompensen los avances, apoyando el desarrollo individual y el de los equipos.

- Desarrollen confianza compartiendo información y conocimiento siempre que sea posible.

\section{Motivar a la gente}

Para que las personas implementen las decisiones con éxito y tengan suficiente segu- ridad para tomar sus propias decisiones, es necesario motivarlas. En un equipo, el líder debe entender con exactitud qué es lo que motiva a sus integrantes a actuar, qué influencias externas están afectándolos y cuál es el papel del líder en el proceso. Hay ocho reglas de motivación:

- Usted mismo debe estar motivado, para ofrecer un ejemplo claro e impulsar el avance.

- Entienda qué es lo que motiva a la gente y elija personas que tengan alta motivación.

- Trate a cada persona como un individuo y evite suponer cosas acerca de ellas.

- Establezca metas realistas y difíciles.

- Recuerde que el progreso incentiva, porque refuerza la seguridad.

- Cree un ambiente motivador. Cómo lo haga dependerá de la tarea y del individuo, pero podría significar, por ejemplo, presionar o estimular a la gente para que actúe, o bien eliminar los obstáculos que le impiden actuar.

- Reconozca el éxito. Esto ayuda a mantener el impulso y contribuye a la continuidad del éxito.

- Dé recompensas justas. Son una forma de reconocimiento y pueden alentar y desarrollar la confianza y el compromiso.

Una visión potente también ayuda a incentivar a la gente. Un buen ejemplo de esto es un comentario de John F. Kennedy, ex presidente de los Estados Unidos: elegimos ir a la luna en esta década y hacer las otras cosas, no porque sean fáciles, sino porque son difíciles. Porque esa meta servirá para organizar y medir lo mejor de nuestra capacidad y de nuestras destrezas, porque es un reto que estemos dispuestos a aceptar, que no deseamos postergar y que pretendemos ganar. 
La gente responde mejor cuando entiende lo que está haciendo. Un líder eficiente debe tener la capacidad de crear y comunicar una visión realista y convincente que sostenga una organización y a sus integrantes tanto en los buenos tiempos como en los malos. Una visión así encapsula una serie de valores que guiarán las decisiones, la acción y desarrollarán la seguridad, el trabajo de equipo y la coherencia.

Además de convincente y realista, una visión debe ser potente si tiene que servir para entusiasmar e inspirar. Debe también ser fácil de comunicar a todos y lo suficientemente específica para ser realmente útil en la toma de decisiones. Debe, además, tener la flexibilidad suficiente para permitir la iniciativa individual y los cambios en las condiciones.

\section{Servir a los clientes y administrar los cambios}

Para una empresa comercial, el conocimiento de sus clientes actuales y potenciales brinda información sobre la cual basar una amplia gama de decisiones. Pero los mercados y los clientes que los componen están constantemente sujetos a cambios. Por lo tanto, es necesario que los clientes entiendan dónde, cómo, cuándo y por qué se producen los acontecimientos para garantizar que las decisiones que toman no sean erróneas ni resulten perjudicados por las circunstancias cambiantes. Hay varias cosas que marcan una diferencia:

- Una visión clara promueve un sentido compartido del objetivo, lo cual hace que resulte más fácil actuar con flexibilidad y adaptarse a las circunstancias cambiantes.

- Es importante garantizar que la burocracia no limite las decisiones ni la necesidad de acción.
- Se deben desarrollar las destrezas de la gente para que pueda hacer frente a los retos impuestos por las circunstancias variables.

- Enfrentar a tiempo los problemas y sus causas originales evita frustraciones $y$ preserva el impulso de cambio.

\section{Comunicar}

Todas las decisiones se deben explicar a quienes se verán afectados por ellas para evitar malentendidos. La explicación debe poner en relieve dónde residen los problemas y las dificultades, como también los beneficios. Debe haber comunicación durante todo el proceso de toma de decisiones a fin desarrollar y mantener el apoyo para la decisión que llegue a tomarse y garantizar que quienes se verán afectados por ella se sientan partícipes.

\section{El manejo de las decisiones críticas}

Decisiones críticas son aquéllas en las que no podemos permitirnos fallar. Pueden ser una parte, quizá vital, de un proceso mayor de una crisis, o simplemente pueden darse en un área de importancia crítica. Un ejemplo de esto fue la crisis de los misiles cubanos en 1962. La Unión Soviética estaba instalando armas nucleares en Cuba, a 150 kilómetros de los Estados Unidos, los que promovió un bloqueo naval de la isla. Las dos superpotencias se hallaban en un impase peligroso. Luego de un tenso estancamiento de las relaciones, el presidente Kennedy recibió un mensaje de Nikita Khrushchev, el líder soviético, que anunciaba el retiro de las armas. A las pocas horas, llegó un segundo mensaje que decía que el retiro quedaba condicionado. Kennedy decidió hacer caso omiso del segundo mensaje. Rápidamente escribió a Khrushchev, aceptando el retiro anunciado 
en la primera carta. Aunque Kennedy no lo sabía entonces, el segundo mensaje había sido enviado en primer lugar. Uno de los resultados de esta crisis fue el establecimiento de un teléfono rojo, un enlace directo entre los líderes de ambos países, para garantizar que jamás se repitieran malentendidos potencialmente tan desastrosos.

\section{Base sus decisiones en la mejor información disponible}

Para tomar la decisión correcta, es necesario estar bien informado. Como dice el ex presidente de la república Argentina, Menen:

Me di cuenta de que uno de los aspectos más importantes del management exitoso es tener una visión objetiva de los hechos. La gente suele equivocarse cuando sus decisiones se basan en un conocimiento inadecuado de los datos disponibles.

Resulta tentador dejar de lado cierta información por obvia o irrelevante, en lugar de tomarse el tiempo para desarrollarla y llegar a una comprensión original.

\section{Sea práctico}

Vuelva a los principios básicos. En lugar de empantanarse en detalles, pregunte: “¿Qué estamos tratando de lograr?”. Esto podrá parecerle trillado, pero a menudo se olvida entre las presiones y los proceso. Las decisiones críticas pueden fracasar porque, aunque parezcan lógicas en teoría, en la práctica son imposibles de realizar.

En 1997, General Motors firmó un acuerdo para un emprendimiento conjunto a largo plazo con pequeño pero potencialmente grande mercado chino de automóviles de alta calidad. Sin embargo, luego de la firma, los chinos introdujeron recortes importantes en los aranceles sobre automóviles y repues- tos importados como parte de su esfuerzo por ingresar a la Organización Mundial del Comercio. Asimismo, el acuerdo requería que GM fabricara el $40 \%$ de sus componentes en China, una cifra que se elevaba al $80 \%$ al tercer ańo. Esto, combinado con la cadena de suministros subdesarrollada de ese país para los fabricantes de automóviles, era una amenaza para el costo y la calidad. Dado que los costos de mano de obra constituían una porción cada vez más reducida del costo general de fabricación de automóviles, eran muy pocos los beneficios que podían obtenerse de uno de los puntos más fuertes de China: su mano de obra. Tras la firma del acuerdo, el mercado global automotor chino se estancó, y las ventas del modelo de alta calidad de GM disminuyeron. Por fin, aunque parecía poco probable que los chinos ofrecieran una licencia a otro fabricante de automotores, el retiro de Peugeot del mercado significó que su licencia quedaba disponible, y Honda, uno de los principales competidores de GM, ganó la licitación.

GM se basó en una visión a largo plazo, suponiendo que su negocio crecería y prosperaría a medida que se expendiera la economía china, y por ende, la demanda. Sin embargo, este caso pone en relieve el peligro de que se agrupen factores externos, lo cual es una amenaza para las decisiones estratégicas y las inversiones importantes. Ilustra la necesidad de tener una comprensión práctica de las cuestiones que intervienen en las grandes decisiones.

\section{Cómo asegurar que las decisiones de liderazgo exitosas. Involucre a la gente en el proceso de toma de decisiones.}

Georges Clemenceau, ex primer ministro francés, dijo en la Conferencia de Versalles en 1919: 
"La guerra es un asunto demasiado serio para dejarla en manos de los generales".

Se dice, a menudo con poca sinceridad, que la gente es el mejor recurso que tiene cualquier organización. Cuando se trata de tomar e implementar decisiones, es la gente la que determina en gran medida el éxito o el fracaso. Por lo tanto, resulta crucial que los empleados estén bien administrados, que se exploten sus ideas, su potencial y que estén motivados y comprometidos.

\section{Sea honesto y ético}

Una empresa debe demostrar su honestidad y su integridad tanto al mundo exterior como a sus propios empleados. Las decisiones que no tienen integridad tienen poca probabilidad de alcanzar el éxito y a menudo resultan perjudiciales. La deshonestidad hace que las decisiones difíciles sean peores o más difíciles de tomar, y a medida que disminuyen la confianza y el respeto de la gente, puede llegar a ser imponible tomar o implementar decisiones futuras.

\section{Entienda que las decisiones sientan precedentes}

Las decisiones pueden sentar precedentes que pueden resultar útiles o bien ser un obstáculo para quienes deban tomar decisiones en el futuro. Tome en cuenta no solo si una decisión sienta precedente o no, sino también si los métodos elegidos para implementarla establecen expectativas para el futuro. Los precedentes pueden resultar útiles para demostrar a otros cómo se toman decisiones, se resuelven problemas y se administra en general. Sin embargo, también pueden establecer las prácticas erróneas como norma.

\section{Demuestre coherencia y apoyo}

Una característica de quienes tienen éxito en la toma de decisiones es su compromiso y su entusiasmo. Estas virtudes suelen engendrar apoyo, pues la gente responde bien a la naturaleza contagiosa del entusiasmo, siempre que no resulte excesivo o inapropiado. Además, a la gente le gusta contribuir, y el entusiasmo a menudo ofrece una oportunidad de brindar ayuda y apoyo.

\section{Implemente las decisiones en forma eficiente}

Las decisiones pueden parecer maravillosas cuando se las analiza en una oficina o en la sala de juntas. Sin embargo, hay tres puntos importantes para recordar:

- Lo que importa es cómo resultará la decisión, o incluso si puede dar resultado. Si una decisión no es práctica, está condenada al fracaso.

- Para ser práctica, una decisión debe ser dinámica. Debe tener suficiente flexibilidad para tomar en cuenta los cambios en las circunstancias.

- Las decisiones estratégicas a menudo necesitan un enfoque paciente y decidido. Lo que importa es qué se propone lograr la decisión, más que la decisión en sí. Esta es apenas un hito en el camino hacia la meta.

\section{Sea metódico}

La toma e implementación de decisiones importantes debe planificarse en forma minuciosa y metódica. La planificación y el monitoreo ayudarán a garantizar que se tomen las medidas correctas en el momento oportuno y evitarán que se acumulen los problemas. Además, un enfoque metó- 
dico permitirá que se complete cada etapa antes de iniciarse la siguiente. Un enfoque demasiado informal, apresurado o poco concentrado probablemente dará resultados insatisfactorios.

\section{Sea positivo y mantenga el sentido del humor}

Aun ante la adversidad, es bueno mantener una actitud positiva. Esto no quiere decir que las decisiones se deban tomar a la ligera, sino que una actitud mental positiva es una fuente importante de fuerza y ventaja. Entre las conductas a evitar en la toma de decisiones se cuentan el pánico y el hábito de postergar las cosas. En cambio, si se adopta un enfoque sereno, positivo y apropiado que revele las cualidades adecuadas en el momento oportuno (como urgencia, precaución, dureza y flexibilidad), aumentan considerablemente las probabilidades de éxito. Siempre enfrente los problemas. Jamás los ignore con la esperanza de que desaparezcan.

\section{Volar alto: fundamentos del liderazgo}

Es bien conocido el valor de un liderazgo fuerte, pero quizás las cualidades que más importan resulten menos obvias. Primero, valorar a la gente. Si a uno no le gusta la gente, debe dedicarse a otra cosa. Es esencial entender a la gente, incentivarla, movilizarla y comunicarse con ella y esto se da especialmente en una empresa de servicios, como lo es una aerolínea. El líder debe ser capaz de descubrir los talentos de la gente. La siguiente cualidad consiste en reducir costos y ser competitivo, pero a la vez tener la certeza de que la gente entiende la estrategia. Si la gente está disconforme o enojada, esto perjudica a la compañía.
En tiempos de preocupación por la calidad de las cuentas financieras y la integridad en la sala de juntas, resulta clara la importancia de un liderazgo fuerte.

Trate de ser transparente, claro y franco. Aun cuando sea difícil, y sobre todo cuando es difícil.

\section{Desarrollar un estilo personal de toma de decisiones}

Para garantizar que su estilo para tomar decisiones sea efectivo, puede resultar útil tomar distancia del proceso y analizar cómo es su enfoque típico. ¿Cuáles son las fortalezas y las debilidades del enfoque? ¿Qué medidas se necesitan para mejorar y desarrollar las destrezas y capacidades en este aspecto? En particular, reflexione acerca de cada etapa del proceso de toma de decisiones y decida en qué áreas se podrían mejorar las destrezas.

Esto significa saber cuándo se puede tomar una decisión en forma independiente y cuándo se necesita apoyo en otros. Significa también saber, por ejemplo, cuándo confiar en los propios instintos, cuando recabar más información y cuando hacer participar a otras personas. Hay muchos otros elementos que son significativos: por ejemplo, cuándo aplicar los principios y cuándo ser pragmático; cuándo hacer concesiones y cuándo mantenerse firme; cuándo ser innovador y desafiante; cuándo adherir a lo establecido; y, sobre todo, prever cuándo una decisión tendrá éxito y cuándo fracasará.

Por medio de este análisis, debería mejorar la calidad de las decisiones, y debería haber también más coherencia, con lo cual será más fácil para los demás entenderlas y emularlas. Asimismo, el desarrollo de un enfoque claro y coherente de la toma de decisiones proporciona una posición de respaldo, de modo que cuando aumenten la presión 
y/o la complejidad o haya más urgencia, se cuente con un enfoque confiable, probado y demostrado, refinado durante tiempos menos estresantes o críticos. Si puedes mantener la cabeza mientras a tu alrededor todos la pierden, es posible que no hayas entendido la situación.

Sin embargo, no hay escape: el rol de quien debe tomar decisiones estratégicas es solitario y debe soportar mucha presión, y a menudo se ve sometido a cierta incertidumbre como riesgo ocupacional que depende del resultado de la decisión. Es importante desarrollar estrategias personales para manejar esta presión; pero, en última instancia, llegar a decisiones que alcanzan el éxito resulta inmensamente gratificante.

\section{CONCLUSIONES}

En momentos cruciales para el Perú, donde tenemos un reto todos los peruanos, necesitamos gente empresarial con una nueva mentalidad gerencial en base a un liderazgo empresarial para poder enfrentar los nuevos retos. Este liderazgo debe basarse en aprender de los errores, analizar las opciones que se presenten en el transcurso del trabajo en equipo, la delegación de funciones es importante hoy más que nunca, los gerentes deben comprender que no se puede avanzar si no se hace participar a los trabajadores, la motivación es importante entre ellos para que comprendan que el servicio a los clientes es factor fundamental, hoy el cliente es el socio estratégico de la empresa. La comunicación y el dialogo debe prevalecer en el líder, el manejo de las situaciones críticas, la información que se acumule es prioritaria para una buena toma de decisiones, el ser práctico, honesto, ético, coherente, metódico, positivo y tener un valor alto ayudará a que el liderazgo sea exitoso.

\section{REFERENCIAS}

Fernández Aguado, Francisco Javier. Liderar, mil consejos para un directivo. Ed. Dossat, 2005.

Hax, Arnoldo C. y Nicholas Majluf. Estrategias para el liderazgo competitivo. Ed. Granica, 2005.

Levy, Alberto. Liderando en el infierno. Competitividad, empresas, clusters y ciudades. Ed. Rústica, 2003.

Palomo Vadillo, María Teresa. Liderazgo y motivación de equipos de trabajo. Ed. Esic, 2005. 